\title{
Including Geometry in Graph Representations: A Quadratic-Time Graph Isomorphism Algorithm and Its Applications
}

\author{
X.Y. Jiang, H. Bunke \\ Dept. of Computer Science, University of Bern \\ Neubrückstrasse 10, CH-3012 Bern, Switzerland
}

\begin{abstract}
In graph representations of objects, geometric information is typically lost. This has forced researchers to use graph matching techniques that are intended to handle general graphs. By encoding the lost geometric information into graph representations, however, we can apply more efficient algorithms for constrained graphs. In this paper we introduce an edge ordering property that is satisfied in many applications. Given this property, the graph isomorphism problem is solvable in quadratic time. We discuss three concrete applications that can be reduced to the graph isomorphism problem and can thus profit from the quadratic-time graph isomorphism algorithm. The improved performance is demonstrated by simulation experiments.
\end{abstract}

\section{Introduction}

Structural description is one of the most general methods for representing the real world and thus very popular in pattern recognition and image analysis. One such structural description are attributed graphs. Using attributed graphs for object representation, the pattern recognition task is casted into that of graph matching. While graphs are able to capture the essential structure of an object, some geometric informations are often lost during the modeling process. This problem is illustrated in Figure 1, where we consider the matching of the model graph of a frustum of pyramid with that of an unknown object. On the object there is a natural ordering of the edges incident to a vertex when we traverse the faces incident to the vertex, for example, clockwise. This important information, however, is no more available in the graph representation of the object. As a result, isomorphisms will be found that are legitimate with regard to the abstract graphs but violate fundamental geometric constraints, i.e., the ordering. One such isomorphism is $\left\{\left(v_{1}, v_{5}^{\prime}\right),\left(v_{2}, v_{6}^{\prime}\right),\left(v_{3}, v_{7}^{\prime}\right),\left(v_{4}, v_{8}^{\prime}\right),\left(v_{5}, v_{1}^{\prime}\right),\left(v_{6}, v_{2}^{\prime}\right),\left(v_{7}, v_{3}^{\prime}\right)\right.$, $\left.\left(v_{8}, v_{4}^{\prime}\right)\right\}$. If we order the vertices connected to $v_{1}$ clockwise, then they build a cycle $\left(v_{2}, v_{5}, v_{4}\right)$. This property should be retained by the corresponding vertices under the above isomorphism, i.e., the vertices $\left(v_{6}^{\prime}, v_{1}^{\prime}, v_{8}^{\prime}\right)$ should also be in clockwise order around $v_{5}^{\prime}$. But this clearly doesn't correspond to the given ordering in the scene graph.

Obviously, the generation of geometrically invalid matchings is caused by the loss of geometric information during the modeling of an object in terms of 

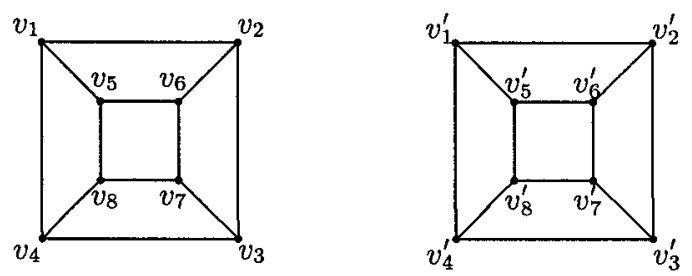

Fig. 1. A model graph (left) and a scene graph (right).

a graph. The problem is not tragic as such. For any graph matching algorithm we can always check the matching results against geometric constraints of the problem domain and then retain only valid matchings. More serious, however, is another problem caused by the loss of geometric information. We are namely forced to use graph matching algorithms for general graphs, mostly variations of branch-and-bound algorithms. These algorithms are computationally very costly. For example, all known algorithms for graph and subgraph isomorphism detection are of exponential complexity. But much more efficient algorithms are available for constrained graphs and their application becomes possible if additional geometric informations are encoded in the graph representations.

In this paper we study a special class of graphs where the edges incident to a vertex have a unique order. In many applications the ordering is naturally derived from the underlying geometry of the patterns, as exemplified by the matching problem in Figure 1. This ordering property ${ }^{1}$ of the graph representation allows the use of an efficient quadratic-time graph isomorphism algorithm. We investigate three concrete applications that can be reduced to solving the graph isomorphism problem, namely weighted graph matching, modeling and classification by random graphs, and generation of characteristic view representations of objects. By means of simulation, we compare the quadratic-time graph isomorphism algorithm with one of the most efficient graph isomorphism algorithms for general graphs. Also, our weighted graph matching method is compared with with other known algorithms that don't make use of the ordering property. The simulation results show clearly the usefulness of the additional geometric information in graph matching.

The rest of this paper is organized as follows. In the next section we introduce the quadratic-time graph isomorphism algorithm. Then, the three applications are described, followed by the simulation results that demonstrate the improved performance by using the quadratic-time graph isomorphism algorithm. Finally, some discussions conclude the paper.

\section{Quadratic-time graph isomorphism algorithm}

In a finite connected graph it is always possible to construct a cyclic directed path passing through each edge once and only once in each direction (see for

\footnotetext{
${ }^{1}$ The same edge ordering information has been used in [9] to resolve ambiguities in the extraction of line drawings.
} 
example [7, p. 41]. A simple way of actually finding such a path is as follows. We call a vertex old or new if it has or has not been reached previously, respectively. An edge being traversed in one direction is termed old if it has been previously traversed in the opposite direction, and new otherwise. In traversing an edge we go from an initial vertex to a terminal vertex. Starting with an edge traversed in one of its directions, we follow these rules:

1. When a new vertex $v_{i}$ is reached from $v_{j}$, then go to vertex $v_{k}$ that is located immediately after $v_{j}$ in the ordered cycle of the vertices connected to $v_{i}$.

2. When an old vertex is reached on a new path, go back in the opposite direction.

3. When an old vertex $v_{i}$ is reached from $v_{j}$ on an old path, then go to the first vertex $v_{k}$ after $v_{j}$ in the ordered cycle of the vertices connected to $v_{i}$ that has not previously been traversed in that direction. (Thus, edges are traversed only once in each direction.)

Now a numbering scheme is introduced to obtain a code. As we reach a new vertex we label it with a new number in natural order. Thus, the label of the initial vertex of the starting edge is 1 , the label of its terminal vertex is 2 , the label of the next new vertex is 3 , a.s.o. We form a code consisting of the numbers of the vertices in the order in which they are visited.

Triply connected planar graphs have a unique embedding in the plane and thus belong to the graph class under our investigation. For this special case Weinberg [13] has proved:

Theorem 1. Let $G_{1}=\left(V_{1}, E_{1}\right)$ and $G_{2}=\left(V_{2}, E_{2}\right)$ be two triply connected plane graphs (graphs embedded in the plane), and $\left(v_{i}, v_{j}\right),\left(v_{i}^{\prime}, v_{j}^{\prime}\right)$ be edges of $G_{1}, G_{2}$, respectively. There exists an isomorphism between $G_{1}$ and $G_{2}$ such that $v_{i}$ and $v_{j}$ correspond to $v_{i}^{\prime}$ and $v_{j}^{\prime}$, respectively, if and only if $C\left(v_{i} v_{j}\right)=C\left(v_{i}^{\prime} v_{j}^{\prime}\right)$.

On the basis of this theorem a simple algorithm for determining all isomorphisms of two triply connected plane graphs was proposed [13]:

1. Choose arbitrarily a directed edge $\left(v_{i}, v_{j}\right)$ of $G_{1}$ and compute $P\left(v_{i} v_{j}\right)$ and $C\left(v_{i} v_{j}\right)$.

2. For each directed edge $\left(v_{i}^{\prime}, v_{j}^{\prime}\right)$ of $G_{2}$ do step 2.1.

2.1. If $C\left(v_{i} v_{j}\right)=C\left(v_{i}^{\prime} v_{j}^{\prime}\right)$ then there exists an isomorphism between $G_{1}$ and $G_{2}$ that maps each vertex in $P\left(v_{i} v_{j}\right)$ to the corresponding vertex in $P\left(v_{i}^{\prime} v_{j}^{\prime}\right)$.

For more efficiency this algorithm can be modified in the following way. If some vertex number during the generation of $C\left(v_{i}^{\prime} v_{j}^{\prime}\right)$ is not identical to the corresponding vertex number in $C\left(v_{i} v_{j}\right)$, we stop the coding process immediately and turn to the next edge of $G_{2}$.

The above theorem can be easily extended to (see [6]):

Theorem 2. Let $G_{1-}=\left(V_{1}, E_{1}\right)$ and $G_{2}=\left(V_{2}, E_{2}\right)$ be two graphs where the edges incident to each vertex have a unique order around the vertex, and $\left(v_{i}, v_{j}\right)$, $\left(v_{i}^{\prime}, v_{j}^{\prime}\right)$ be edges of $G_{1}, G_{2}$, respectively. There exists an isomorphism between $G_{1}$ and $G_{2}$ such that $v_{i}$ and $v_{j}$ correspond to $v_{i}^{\prime}$ and $v_{j}^{\prime}$, respectively, if and only if $C\left(v_{i} v_{j}\right)=C\left(v_{i}^{\prime} v_{j}^{\prime}\right)$. 
According to this theorem, the isomorphism detection algorithm given above can also be used to handle the class of graphs under investigation in this paper.

The computational complexity of this algorithm is of order $O\left(m^{2}\right)$ in the worst case where $m$ is the number of edges of the graphs. Since the worst case occurs only in the case of many automorphisms of the graphs, the average computational expense can be expected substantially better than quadratic. A performance evaluation of this method as compared with one of the most efficient graph isomorphism algorithms for general graphs will be given in Section 6.1.

It is worth mentioning that in the literature there exist other low-order polynomial isomorphism algorithms for planar graphs, see for example [4, 5]. But these algorithms are either so complicated that no actual implementation is known to date, or are non-constructive. Moreover, the restrictions to planar graphs is too strong and there is no easy way to extend them to the class of graphs considered in this paper.

\section{$3 \quad$ Weighted graph matching}

Let $G_{1}=\left(V_{1}, E_{1}, w_{1}\right)$ and $G_{2}=\left(V_{2}, E_{2}, w_{2}\right)$ be two weighted graphs with $n$ vertices where $w_{k}: V_{k} \times V_{k} \rightarrow R$ is a weight function of real values for edges $\left(v_{i}, v_{j}\right)$ and vertices $\left(v_{i}, v_{i}\right) \equiv v_{i}$. The weighted graph matching problem (WGMP) is to find an one-to-one mapping $\Phi$ between $V_{1}=\left\{v_{1}, v_{2}, \cdots, v_{n}\right\}$ and $V_{2}=$ $\left\{v_{1}^{\prime}, v_{2}^{\prime}, \cdots, v_{n}^{\prime}\right\}$ so that a difference measure $F(\Phi)=\sum_{i=1}^{n} \sum_{j=1}^{n} f\left(w_{1}\left(v_{i}, v_{j}\right)-\right.$ $\left.w_{2}\left(\Phi\left(v_{i}\right), \Phi\left(v_{j}\right)\right)\right)$ is minimized where $f(\cdot)$ is an application specific difference function.

A number of approaches to solving the WGMP have been proposed. Yang [17] and You [18] employed tree search techniques of exponential time complexity. Their methods always give the true optimum solution. For the difference function $f(\cdot)=(\cdot)^{2}$ Umeyama [11] proposed a polynomial time analytical method based on the eigendecomposition of the adjacency matrix of a graph. An improved version of this algorithm with increased computation time is described in [3]. With this technique the true optimum solution is obtained only when the weights of the two graphs are sufficiently close to each other. For another difference function $f(\cdot)=|\cdot|$ Almohamad [1] suggested a linear programming approach to the WGMP. All methods in $[1,3,11]$ may fail to find the optimum matching. Also, it is not possible to incorporate any structural information like the ordering of edges considered in this paper. As a consequence, a geometrically invalid optimum matching may result. This type of failure is demonstrated in Figure 2. For $f(\cdot)=(\cdot)^{2}$, the structure-preserving optimum matching should be $\left\{\left(v_{1}, v_{1}^{\prime}\right),\left(v_{2}, v_{2}^{\prime}\right),\left(v_{3}, v_{3}^{\prime}\right),\left(v_{4}, v_{4}^{\prime}\right)\right\}$. However, the solution found by Umeyama's algorithm is $\left\{\left(v_{1}, v_{1}^{\prime}\right),\left(v_{2}, v_{3}^{\prime}\right),\left(v_{3}, v_{2}^{\prime}\right),\left(v_{4}, v_{4}^{\prime}\right)\right\}$ with a zero difference.

For the special graph class under consideration we suggest the following method for the WGMP. We apply the quadratic-time graph isomorphism to find all isomorphisms between $G_{1}$ and $G_{2}$. For each isomorphism $\Phi$ we compute the difference function $F(\Phi)$ and choose the $\Phi$ with minimum $F(\Phi)$ as the solution to the WGMP. For more efficiency we could also proceed in an alter- 

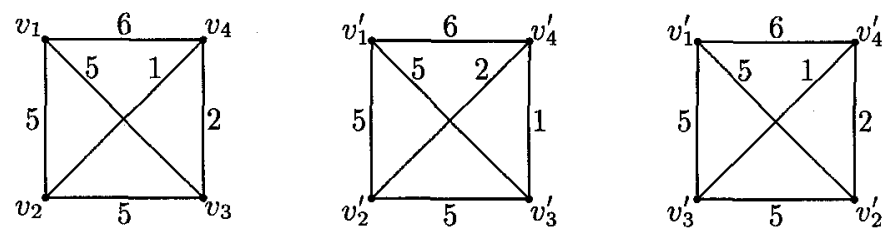

Fig. 2. The optimum matching found by Umeyama's algorithm for the two weighted graphs (left and middle) is shown on the right.

native way. If during the path generation process the difference measure of the partial path generated so far becomes larger than that of the best matching already found, then we can stop immediately and turn to the next path. In both cases the matching found is guaranteed to be geometrically consistent. However, the active utilization of the ordering information makes a drastic reduction of the computation expense possible. Also, the number of possible isomorphisms is drastically reduced. While isomorphisms may be of exponential order in general, there are at most $2 m$ geometrically valid isomorphisms if we match two graphs of $m$ edges each under consideration of the ordering property. Compared with the algorithms in $[1,3,11]$ our method has an additional highly desirable advantage besides the structure-preserving property. While only numerical attributes are allowed there, we are able to use any type of attributes, including symbolic attributes and multiple attributes of mixed types. In our method the attributes may be defined differently for the vertices and edges of a graph, Moreover, an arbitrary difference measure can be handled. A performance evaluation of our method as compared with some other algorithms will be given in Section 6.2.

\section{Modeling and classification by random graphs}

When uncertainty exists in structural patterns due to noise or deformations, a probabilistic description of the pattern ensemble is necessary. For this purpose Wong et al. $[14,15]$ have proposed the concept of random graph. To capture the variability of attributes in the attributed graphs corresponding to the training samples of the same class, both vertices and edges are considered as random variables that can take different values from a definition domain with some probability. Then, the attributed graph of a concrete sample is only a specific outcome of the random graph.

In the learning phase the random graph of a pattern class is acquired through the synthesis of attributed graphs of the training samples where isomorphisms must be determined. Suppose that random graphs $R_{1}, R_{2}, \cdots R_{n}$ represent $n$ different pattern classes and $G$ is the attributed graph of an unknown pattern. To classify $G$ we need again a graph isomorphism test between $G$ and $R_{i}$ so that the probability $P\left(G, R_{i}\right)$ of $G$ being an outcome of $R_{i}$ is obtained. Using the maximum likelihood rule, $G$ is assigned to class $k$ iff $p\left(R_{k}\right) p\left(G, R_{k}\right)>$ $p\left(R_{i}\right) p\left(G, R_{i}\right), i \neq k$, where $p\left(R_{i}\right)$ is the a priori probability of class $i$ characterized by the random graph $R_{i}$. 
Important for our discussion here is the fact that the graph isomorphism problem must be solved in the learning and recognition phase. Any tree search technique can be used for this purpose. In [8] a more specific tree search algorithm is suggested that makes explicit use of the characteristics of random graphs. As far as the ordering property can be defined for the random graphs under investigation, the quadratic-time graph isomorphism algorithm described in Section 2 is applicable to this problem, too. This way both learning and classification can be performed substantially faster than by using a tree search technique.

\section{Generation of characteristic views of objects}

Characteristic view (CV) representation ${ }^{2}$ is one of the most important object representations and has found applications in object recognition and computer graphics [2]. In the viewing space a view of an object becomes a line drawing obtained by projecting all the (partially) visible edges of the object onto the image plane. The graph structure of the view depends on where the viewpoint is located relative to the object. Although there exist an infinite number of viewpoints, the entire viewing space can be partitioned into a finite number of regions, called characteristic view domains, so that all views within a region have identical graph structure and represent thus a characteristic view of the object. It is possible that the views from two different $\mathrm{CV}$ domains of an object are equivalent. In this case the concept of characteristic view class is introduced. Therefore, the number of $\mathrm{CV}$ classes may be less than the number of $\mathrm{CV}$ domains. For instance, a cube has $26 \mathrm{CV}$ domains but only $3 \mathrm{CV}$ classes with one, two and three visible faces, respectively [12].

One popular way of generating the CV representation of an object makes use of a quasi-uniform tesselation of the viewing space. A view of the object is created for each sample point of the tesselation and adjacent equivalent views are grouped together to form $\mathrm{CV}$ domains. At the next level equivalent CVs from nonadjacent CV domains are grouped together to form CV classes. Therefore, checking whether two given views are equivalent is a fundamental operation in the generation of $\mathrm{CV}$ object representations. This process is a graph isomorphism check $^{3}$. Typically, tree search techniques of exponential time complexity are used for this purpose [12].

Generation of the CV representations of objects provides an excellent example problem where we can explore the ordering property of graph structures. The graph representation of a view is an embedded plane graph, i.e., a planar

${ }^{2}$ Terms like aspect graph, characteristic view, principal view and stable view have been synonymically used in the literature. In this paper we follow the terminology in [12].

${ }^{3}$ Actually, the equivalence check also contains a geometric matching to ensure that the two views are related to each other by a $3 \mathrm{D}$ geometric transformation. This geometric matching is usually casted into a comparison of symbolic and numeric attributes $[12]$ to be done in addition to the graph isomorphism algorithm. 


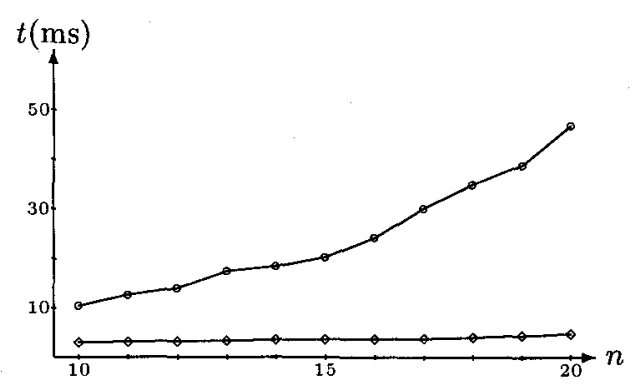

(a)

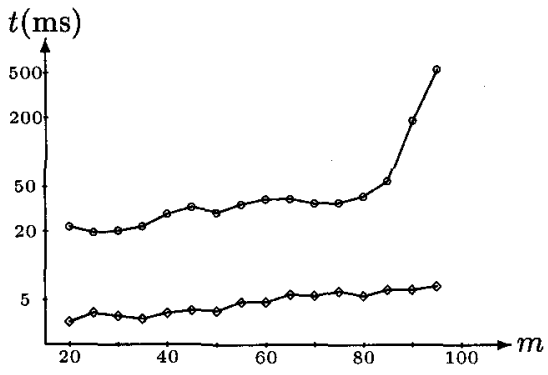

(b)

Fig. 3. Comparison of graph isomorphism algorithms. The symbols $\diamond$ and o stand for the quadratic-time and Ullmann's algorithm, respectively.

graph with a fixed embedding in the plane. Consequently, the ordering information is available in the views in a natural way. Instead of expensive tree search techniques we can thus use the substantially more efficient quadratic-time graph isomorphism algorithm described in Section 2. The applicability of efficient graph isomorphism algorithms has not been recognized in earlier works on the generation of CV representations of objects. Although this task is done in an off-line phase for applications in both object recognition and computer graphics, we believe that the use of the quadratic-time graph isomorphism algorithm is still very attractive due to its efficiency and simplicity.

\section{Performance evaluation}

Simulation experiments are presented here to illustrate the performance of the quadratic-time graph isomorphism algorithm and its application to the weighted graph matching problem as compared with a number of other methods.

\subsection{Graph isomorphism}

We have compared the quadratic-time graph isomorphism algorithm with one of the most efficient graph isomorphism algorithms developed by Ullmann [10] for general graphs. For each of a variety of graph configurations $(n, m), 50$ pairs of random graphs with $n$ vertices and $m$ edges are generated and matched to each other to find all isomorphisms. Here the second graph of a pair is created by shuffling the number of vertices. The edges incident to a vertex are assumed to be in a particular order and this order is retained in the second graph of the pair. The ordering information is used in the quadratic-time algorithm but not in Ullmann's method.

As representative for the whole simulation series, Figure 3(a) shows the average computation time for each graph pair on a SUN Sparcstation 5 for $n \in[10 . .20]$ und $m=2 n$. For $n=15$, the behavior of both algorithms for different $m$ values is illustrated in Figure 3(b) with a logarithmic scale. As the graph 


\begin{tabular}{|c|c|c|c|c|c|c|c|c|c|}
\hline \multicolumn{4}{|c|}{$n=10$} & \multicolumn{4}{c|}{$n=15$} \\
\hline \multirow{3}{*}{$\epsilon$} & \multicolumn{2}{|c|}{ our method } & eigen method & & \multicolumn{3}{c|}{ our method } & \multicolumn{2}{c|}{ eigen method } \\
\cline { 2 - 10 } & mean (S.D.) & $k$ & mean (S.D.) & $k$ & $\epsilon$ & mean (S.D.) & $k$ & mean (S.D.) & $k$ \\
\hline \hline 0.00 & $0.00(0.00)$ & 50 & $0.00(0.00)$ & 50 & 0.00 & $0.00(0.00)$ & 50 & $0.00(0.00)$ & 50 \\
\hline 0.05 & $0.04(0.01)$ & 50 & $0.19(0.74)$ & 47 & 0.05 & $0.09(0.01)$ & 50 & $0.32(0.97)$ & 47 \\
\hline 0.10 & $0.15(0.02)$ & 50 & $1.08(1.76)$ & 36 & 0.10 & $0.34(0.03)$ & 50 & $2.99(3.42)$ & 26 \\
\hline 0.15 & $0.34(0.05)$ & 50 & $2.51(2.53)$ & 24 & 0.15 & $0.79(0.07)$ & 50 & $9.26(5.04)$ & 6 \\
\hline 0.20 & $0.59(0.07)$ & 50 & $4.58(2.41)$ & 7 & 0.20 & $1.41(0.12)$ & 50 & $13.57(4.41)$ & 1 \\
\hline
\end{tabular}

Table 1. Performance of two weighted graph matching methods.

\begin{tabular}{|c||c|c|c|c|c|c|c|}
\hline $\boldsymbol{\epsilon}$ & $n$ & 10 & 11 & 12 & 13 & 14 & 15 \\
\hline \hline \multirow{3}{*}{0.05} & our method & 5 & 6 & 7 & 9 & 12 & 16 \\
\cline { 2 - 8 } & eigen method & 10 & 12 & 15 & 18 & 21 & 25 \\
\cline { 2 - 8 } & $\mathrm{A}^{*}-1$ & 119 & 203 & 328 & 530 & 802 & 1268 \\
\cline { 2 - 8 } & $\mathrm{A}^{*}-2$ & 424 & 649 & 958 & 1337 & 1842 & 2516 \\
\hline \multirow{3}{*}{0.1} & our method & 5 & 6 & 7 & 10 & 12 & 15 \\
\cline { 2 - 8 } & eigen method & 10 & 11 & 15 & 19 & 22 & 27 \\
\cline { 2 - 8 } & $\mathrm{A}^{*}-1$ & 423 & 1008 & 2450 & 5074 & 10582 & 21380 \\
\cline { 2 - 8 } & $\mathrm{A}^{*}-2$ & 470 & 832 & 1335 & 2430 & 4686 & 8656 \\
\hline
\end{tabular}

Table 2. Computation time (milliseconds) of four weighted graph matching methods.

gradually becomes almost complete $(m \geq 85)$, the computation time required by Ullmann's method increases drastically while the quadratic-time algorithm remains very fast. The simulation results clearly demonstrate the advantage of utilizing ordering information in graph isomorphism tests. Specifically, they also show the potential of speedup in the generation of characteristic views of objects.

\subsection{Weighted graph matching}

Our weighted graph matching method has been compared with Umeyama's eigendecomposition approach and two branch-and-bound algorithms. We have constructed similar simulation experiments as in [11]. Pairs of weighted complete graphs with $n=10$ to 15 vertices are generated. Random weights in the range $[0.0 . .1 .0]$ are assigned to the first graph $G_{1}$ of a pair. Then, the second graph $G_{2}$ is created by adding uniformly distributed noise in the range $[-\epsilon . . \epsilon](\epsilon \leq 0.2)$ to each weight of $G_{1}$ and shuffing the number of vertices. For each configuration $(n, \epsilon), 50$ pairs of weighted graphs are generated and matched with each other by our method, the eigendecomposition method, the $\mathrm{A}^{*}$ algorithm with the lowerbounded estimate of future costs set to zero $\left(A^{*}-1\right)$, and the $A^{*}$ algorithm with a cost function suggested by Wong [16] $\left(\mathrm{A}^{*}-2\right)$.

As representative for the whole simulation series, Table 1 shows the results for $n=10,15$ and five different noise levels. Here mean and S.D. give the sample 
mean and the standard deviation of the difference function $F(\Phi)$ over 50 trials, respectively. The number $k$ counts the optimum matchings obtained by each algorithm. The results for the two tree search algorithms are identical with that of our method and thus not tabulated. Notice that our algorithm is guaranteed to always yield the correct solution. From Table 1, we can see that Umeyama's method works correctly only for $\epsilon=0.0$. With increasing noise, more and more optimal matchings are missed. For the noise levels $\epsilon=0.05,0.1$ and different sizes of graphs the average computation time for each graph pair is reported in Table 2. In all the simulations our algorithm runs substantially faster than all other methods. While the algorithms described in $[1,3]$ have better performance with respect to the number of optimum matchings than the eigendecomposition method, they are computationally more expensive. The method in [1], for instance, needs typically 50 to 100 times more computation time as reported by the authors. As can be expected, the two tree search algorithms find all optimum matchings at the price of an extremely high computation burden. The simulation results have also shown the well-known fact that a more sophisticated cost function in the $\mathrm{A}^{*}$ algorithm doesn't necessarily reduce the computation time in all cases. Interestingly, the computational expense of the four algorithms depends on quite different factors. The analytical eigendecomposition approach operates on the adjacency matrices of two graphs and its computational performance is thus only a function of the number of vertices of the graphs. For a given graph size, on the other hand, the computation time of our method also depends on the number of edges and the actual structure of the graphs. The situation is more complicated in the tree search algorithms. For the same graph structure they vary even with the actual weights. This phenomenon can be easily observed in the computation time for the two different noise levels in Table 2.

The simulation results clearly demonstrate the advantage of utilizing ordering information in graph matching. In our weighted graph matching method optimum matchings are obtained at an extremely low cost. The proposed algorithm is about twice as fast as the analytical eigendecomposition approach.

\section{Conclusion}

For many applications the full power of matching techniques for general graphs is not really necessary. By encoding additional geometric information into graph representations - in our case the ordering property - we can use more efficient algorithms for constrained graphs. In this paper we have discussed the use of quadratic-time graph isomorphism in three applications. In particular, we have pointed out the intrinsic applicability of efficient graph isomorphism algorithms in the generation of characteristic view representations of objects. By means of simulations we have shown that utilization of the ordering property leads to much more efficient graph isomorphism tests. Also, we have demonstrated that our weighted graph method has a substantially better performance than other known methods. We believe that the idea of bringing geometric informations lost in the graph modeling process back into graph representations is of general interest and will find its use in many other applications. 


\section{Acknowledgments}

We want to thank B. Messmer for providing us his program for Ullmann's algorithm and the two branch-and-bound algorithms used in our simulations.

\section{References}

1. H.A. Almohamad and S.O. Duffuaa, A linear programming approach for the weighted graph matching problem, IEEE Trans. on PAMI, 15(5), 522-525, 1993.

2. K.W. Bowyer, C.R. Dyer, Three-dimensional shape representation, in T.Y. Young (Ed.), Handbook of Pattern Recognition and Image Processing: Computer Vision, Academic Press, 17-51, 1994.

3. M. Hanajik, F.J. Kylstra, R.G. van Vilet, An analytical approach to the matching of attributed graphs, Proc. of Scand. Conf. on Image Analysis, 419-425, 1993.

4. J.E. Hopcroft, R.E. Tarjan, A $V \log V$ algorithm for isomorphism of triconnected planar graphs, Journal of Computer and System Sciences, 7, 323-331, 1973.

5. J.E. Hopcroft, J.K. Wong, Linear time algorithm for isomorphism of planar graphs, Proc. of 6th Annual ACM Symposium on Theory of Computing, 172-184, 1974.

6. X.Y. Jiang, H. Bunke, A simple and efficient algorithm for determining the symmetries of polyhedra, CVGIP: GMIP, 54(1), 91-95, 1992.

7. O. Ore, Theory of graphs, Amer. Math. Soc., Providence, RI, 1962.

8. D.S. Seong, Y.K. Choi, H.S. Kim, K.H. Park, An algorithm for optimal isomorphism between two random graphs, Pattern Recognition Letters, 15, 321-327, 1994.

9. R. Shapira, H. Freeman, A cyclic-order property of bodies with tree-face vertices, IEEE Trans. on Computers, 26(10), 1035-1039, 1977.

10. J.R. Ullmann, An algorithm for subgraph isomorphism, JACM, 23(1), 31-42, 1976.

11. S. Umeyama, An eigendecomposition approach to weighted graph matching problems, IEEE Trans. on PAMI, 10(5), 695-703, 1988.

12. R. Wang, H. Freeman, Object recognition based on characteristic view classes, Proc. of Int. Conf. on Pattern Recognition, Vol.I, 8-12, 1990.

13. L. Weinberg, A simple and efficient algorithm for determining isomorphism of planar triply connected graphs, IEEE Trans. on Circuit Theory, 13(2), 142-148, 1966.

14. A.K.C. Wong, M.L. You, Entropy and distance of random graphs with application to structural pattern recognition, IEEE Trans. on PAMI, 7(5), 599-609, 1985.

15. A.K.C. Wong, J. Constant, M.L. You, Random graphs, in H. Bunke, A. Sanfeliu (Eds.), Syntactic and Structural Pattern Recognition - Theory and Applications, World Scientific, 197-234, 1990.

16. A.K.C. Wong, M. You, S.C. Chan, An algorithm for graph optimal monomorphism, IEEE Trans. on System, Man, and Cybernetics, 20(3), 628-636, 1990.

17. H. Yang, J.-W. Tai, On isomorphisms of attributed relational graphs for pattern analysis and a new branch and bound algorithm, Proc. of Int. Conf. on Pattern Recognition, 957-959, 1988.

18. M. You, A.K.C. Wong, An algorithm for graph optimal isomorphism, Proc. of Int. Conf. on Pattern Recognition, 316-319, 1984. 